\section{Teilnahme von asthmakranken Kindern am Schulsport} Eine Erhebung an Hamburger Schulen

\author{
A. Meyer $^{1}$ \\ M. A. Machnick ${ }^{1}$ \\ W. Behnke ${ }^{1}$ \\ K.-M. Braumann²
}

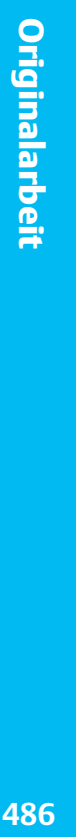

\author{
Participation of Asthmatic Children in Gymnastic Lessons at School
}

\section{Zusammenfassung}

Einleitung: Körperliche Aktivität ist ein prädominanter Stimulus für bronchiale Obstruktion bei Kindern mit Asthma. Deshalb haben Ärzte, Lehrer und Eltern immer noch eine negative Haltung gegenüber der Teilnahme asthmakranker Kinder am Schulsport. Wir untersuchten die Teilnahme asthmakranker Jungen und Mädchen am Schulsport in Hamburg. Methodik: 254 Lehrer, die an 50 verschiedenen allgemeinbildenden staatlichen und nichtstaatlichen Schulen in Hamburg das Fach Sport unterrichten, wurden nach ihren Kenntnissen über diese Erkrankung und nach der Häufigkeit sowie der Art der Teilnahme asthmatischer Kinder am Schulsport befragt. Ergebnisse: Die Lehrer gaben an, dass bei einer durchschnittlichen Klassengröße von 25-30 Schülern im Mittel ein Kind pro Klasse an einem Asthma bronchiale erkrankt ist. $60 \%$ dieser Asthmatiker nehmen genau wie ihre gesunden Mitschüler am Schulsport teil. 40\% nehmen entweder nur zeitweise $(12,6 \%)$, teilweise $(25,2 \%)$ oder aber gar nicht $(12,2 \%)$ teil. Die meisten Lehrer schätzten hierbei ihr Wissen über Asthma als mittel bis gut ein. Im Gegensatz hierzu hatte der Sportunterricht aber in nur 4,7\% einen spezifischen Stundenaufbau, der es den Asthmatikern erlaubt, ohne Einschränkung der Atmung teilzunehmen. Schlussfolgerung: Nicht alle Kinder, die an einem Asthma leiden, sind auch als solche bekannt. Kinder mit Asthma bronchiale sind in der Schule unterfordert und nehmen in nur 60\% gleichberechtigt zu ihren gesunden Mitschülern am Sportunterricht teil, was darauf hindeutet, dass das Wissen über diese im Kindesalter am häufigsten vorkommende Erkrankung nicht ausreichend ist. Die Integration in den Sportunterricht könnte verbessert werden. Dies verlangt jedoch eine bessere Ausbildung der Lehramtsstudenten und Lehrer.

\section{Abstract}

Introduction: Exercise is a predominant stimulus leading to bronchial obstruction in asthmatic children. Therefore physicians, teachers and parents still have a negative attitude towards physical exercise of asthmatic children in school. We investigated the attendance of girls and boys with asthma during sportlessons at school. Methods: 254 teachers who give lessons in sports in 46 different schools (high school, primary and secondary school) in Hamburg were asked about their knowledge of the disease and how often and in which manner asthmatic children participated their sport-lessons. Results: Teachers estimated that in each class (average: 25 - 30 children) approximately 1 asthmatic is present. $60 \%$ of these asthmatics take part likewise their healthy peers. $40 \%$ either take part only sometimes $(12,6 \%)$ or specific elements $(25,2 \%)$ or not at all $(12,2 \%)$. Most of the teachers assessed their knowledge about the disease as medium good. In contrast, only $4,7 \%$ of them had a specific plan that allows asthmatics to participate sport-lessons without getting obstructive. Conclusion: Asthmatic children are underestimated in school and participate in only $60 \%$ equally to their healthy peers at sport-lessons. The knowledge about this most frequent chronic disease in children could be increased. Better training of teacher-students and teachers is recommended.

${ }^{1}$ Universitätsklinikum Hamburg-Eppendorf, Klinik und Poliklinik für Innere Medizin (Leiter: Prof. Dr. med. H. Greten)

${ }^{2}$ Institut für Sport- und Bewegungsmedizin, Fachbereich Sportwissenschaft, Universität Hamburg

Korrespondenzadresse

Dr. med. A. Meyer · Universitätsklinikum Hamburg-Eppendorf · Klinik und Poliklinik für Innere Medizin · Bereich Pneumologie · Martinistr. 52 20246 Hamburg 
Mit einer Prävalenz zwischen 7 und 10\% [1], in Australien sogar von 17,1\% [2], mit weiter steigender Tendenz [3], handelt es sich beim Asthma bronchiale um die häufigste chronische Erkrankung im Kindes- und Jugendalter [1]. Trotz zunehmender Beachtung des Asthma in den letzten Jahren [4] sowie des besseren Verständnisses pathophysiologischer Zusammenhänge [5] und daraus resultierender adäquater Behandlungsmöglichkeiten [6] ist noch immer eine ansteigende Mortalität und Morbidität zu verzeichnen $[5,7]$.

Entsprechend der Häufigkeit der Erkrankung sind zunehmend auch Schule und Lehrer mit der Betreuung asthmakranker Kinder im Unterricht konfrontiert [8].

Besonderheiten im täglichen Umgang mit unter Asthma bronchiale leidenden Kindern und Jugendlichen ergeben sich im Allgemeinen durch erforderliche medizinische und präventive Maßnahmen als auch durch die Berücksichtigung psychosozialer Komponenten sowie der Frage der Lebensqualität [9].

So kommt es bei einem Großteil der Betroffenen zu erheblichen Schulausfallzeiten mit hiermit verbundenen Schuldefiziten [10]. Studien belegen weiterhin [11], dass auch die Inzidenz psychosozialer Probleme 2-3-mal höher ist als bei gesunden Kindern und Jugendlichen [9]. Neben einem Minderwertigkeitsgefühl, Unsicherheit, Abhängigkeit, Ängstlichkeit und Depressivität werden häufig auch Separation und soziale Ausgrenzung beschrieben [12], insofern es nicht zur Ignoration der Erkrankung und somit zu einem non-compliant-Verhalten führt [13].

Körperliche Bewegung und Sport kann diesen Kindern helfen, integriert und akzeptiert zu werden und darüber ein Selbstwertgefühl, Selbstdisziplin und ein Gesamtwohlbefinden aufzubauen, was in einigen Studien bereits vor einigen Jahren belegt werden konnte $[14,15]$.

Da jedoch körperliche Aktivität einen dominanten Stimulus für die bronchiale Obstruktion mit resultierender Dyspnoe bei hyperreagiblen Atemwegen des Asthmatikers darstellt [16], 90\% aller Asthmatiker erleiden Anstrengungsasthma, haben Therapeuten, Lehrer und Eltern häufig eine negative Einstellung gegenüber körperlicher Anstrengung bei Kindern mit Asthma, insbesondere auch was den Schulsport anbelangt. Aus diesem Grund nimmt ein großer Prozentsatz dieser Kinder mit chronischer Erkrankung nicht am Schulsport teil [16].

Ziel dieser Studie war es, die Teilnahme von Kindern und Jugendlichen mit Asthma am Schulsport in Hamburg zu untersuchen sowie den Wissensstand von Lehrern bezüglich des Krankheitsbildes Asthma bronchiale zu erfassen.
Über die Behörde für Jugend, Arbeit und Soziales/Amt für Schule wurden zunächst alle im Jahr 1998/99 existierenden allgemeinbildenden staatlichen und nichtstaatlichen Schulen in Hamburg ermittelt, wobei Sonder- und Förderschulen aufgrund differierender Sportprogramme mit psychomotorischen Komponenten nicht berücksichtigt wurden.

Aus der sich ergebenden Anzahl von 496 Hamburger Schulen (236 Grundschulen, 67 Hauptschulen, 15 Haupt- und Realschulen, 56 Realschulen, 74 Gymnasien, 48 Gesamtschulen) wurde dann der jeweilige prozentuale Anteil der Schulformen pro politischem Bezirk ermittelt (HH-Mitte, Altona, Eimsbüttel, Nord, Wandsbek, Bergedorf, Harburg), um so einen repräsentativen Querschnitt durch das gesamte Hamburger Stadtgebiet einbeziehen zu können.

Ebenfalls unter Berücksichtigung der einzelnen Schulformen und Bezirke wurden hieraus exemplarisch 50 Schulen in die Studie einbezogen: 24 Grundschulen, 6 Hauptschulen, 3 Haupt- und Realschulen, 6 Realschulen, 7 Gymnasien, 4 Gesamtschulen.

Zunächst erfolgte eine telefonische Kontaktaufnahme mit den Schulen, um das Einverständnis der Schulleitung zur Teilnahme an dieser Studie einzuholen und die Anzahl der Sport unterrichtenden Lehrer zu ermitteln.

Insgesamt wurden 440 Fragebogen an die Schulen versandt und mit einem Freiumschlag versehen, um den Rücklauf zu verbessern. Es wurden alle Sportlehrer bzw. die sportunterrichtenden Lehrer anderer Fachbereiche befragt. Eine gesonderte Auswahl nach Klassen und Altersstruktur der Schüler erfolgte nicht. (Abb. 1).

Neben der Erfassung persönlicher Daten des Lehrers wie Geschlecht, Alter, Berufsjahre und Ausbildung bezogen sich 3 Fragen auf den Informations- bzw. Wissensstand über Asthma. Auf einer Skala von gar nicht bis vollständig konnte angegeben werden, wie der eigene Wissensstand eingeschätzt wurde, ob das Wissen aufgrund eigener Erkrankung, Asthmatikern in der Familie oder durch die Ausbildung bzw. andere Quellen erworben wurde und ob eine Zusatzausbildung in Bezug auf Asthma vorlag.

Weitere 6 Fragen bezogen sich auf die Teilnahme asthmatischer Kinder am Schulsport.

Hierbei war von Relevanz, ob und wie viele Kinder mit Asthma bronchiale durchschnittlich eine Klasse besuchten und ob diese Kinder am Sportunterricht teilnahmen.

Ferner wurde erfragt, ob diese Teilnahme gleichberechtigt, nur zu bestimmten Teilen oder zeitweise erfolgte oder ob aufgrund eines Attestes bzw. des Eltern-/Lehrerwunsches die Teilnahme entfiel.

Von Interesse war auch, ob eine Benotung (wie bei allen anderen, gar nicht oder gesondert) bei Teilnahme durchgeführt wurde, ob evtl. ein spezieller Sportunterrichtsaufbau zugrunde lag und ob insgesamt die Thematisierung von Asthmasport während der Ausbildung für sinnvoll erachtet wird. 


\section{Fragebogen für Sportlehrer}

$\begin{array}{llr}\text { Geschlecht: } & \mathrm{m} \square \mathrm{w} \square & \text { Alter } \\ \text { Ausbildung: } & \text { LOA } \square & \begin{array}{r}\text { Grundschule } \\ \text { Sport als Fach }\end{array} \\ & & \square \\ & & \text { kein Sportstudium }\end{array}$

Sind Sie über die Erkrankung Asthma bronchiale informiert?

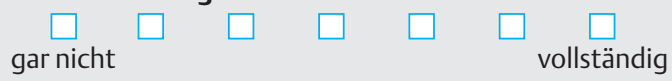

Woher haben Sie ihr Wissen über Asthma?

selber betroffen $\square \quad$ Angehörige mit Asthma $\square \quad$ Ausbildung $\square$

andere Quelle:

Berufsjahre

Grund- und Mittelstufe

Sport als Lernbereich $\square$

Sport als Fortbildung

Haben oder hatten Sie asthmatische Kinder im Sportunterricht? $\quad$ ja $\square \quad$ nein $\square$

Wenn ja, im Durchschnitt pro Klasse: $1 \square \quad 2 \square \quad 3 \square \quad 4 \square \quad 5 \square \quad ; 1-2 \square \quad 2-4 \square$

Nehmen/nahmen die asthmatischen Kinder am Sportunterricht teil? ja $\square$ nein $\square$

Wenn ja: gleichberechtigt $\square \quad$ nur best. Teile des U. $\square \quad$ zeitweise $\square$

Wenn nein: $\quad$ Attest $\square \quad$ Elternwunsch $\square \quad$ Lehrerwunsch $\square$

Wie benoten Sie die Leistungen der asthmatischen Schüler/-innen?

gar nicht $\square \quad$ wie alle anderen $\square \quad$ Sonderbenotung $\square$

Hat Ihr Sportunterricht einen speziellen Aufbau (für die asthmatischen Schüler/-innen)?

ja $\square \quad$ nein $\square$

Haben Sie eine Zusatzausbildung in Bezug auf Asthma?

ja $\square \quad$ nein $\square$

welche?

\section{Ergebnisse}

Von insgesamt 440 Fragebogen, die an 50 Schulen im gesamten Hamburger Stadtgebiet unter Berücksichtigung einzelner Schulformen und Bezirke verschickt wurden, wurden 254 auswertbare Bogen zurückgeschickt; 4 Schulen konnten aufgrund fehlender Beantwortung nicht in die Studie einbezogen werden. Insgesamt entsprach dies einem Rücklauf von 65,8\%.

Die Auswertung der Fragebogen bzw. die Darstellung der Ergebnisse lässt sich in 3 Komplexe unterteilen. Die Teilnahme der unter Asthma bronchiale leidenden Kinder und Jugendlichen am Sportunterricht, der Wissensstand der Lehrer und die Erfassung der persönlichen Daten der Lehrkräfte.

Betrachtet man die Häufigkeit asthmatischer Kinder im Sportunterricht, so zeigte sich, dass $18,5 \%$ der befragten Lehrer keine erkrankten Kinder bzw. 10,2\% < 1 Kind pro Klasse im Unterricht betreuten. 2,8\% beantworteten diese Frage nicht.

Die Verteilung der Häufigkeiten kann dem folgenden Diagramm entnommen werden (Abb. 2).

Auffällig ist, dass bei einer mittleren Klassengröße von 25-30 Schülern $42,4 \%$ der Lehrer durchschnittlich 1 bzw. 12,6\% der Lehrer durchschnittlich 1-2 unter Asthma bronchiale leidende Kinder betreuen, was einer Inzidenz von 3-5\% entsprechen würde.

Die Beteiligung dieser Kinder am Sport wurde mit 74,4\% angegeben.

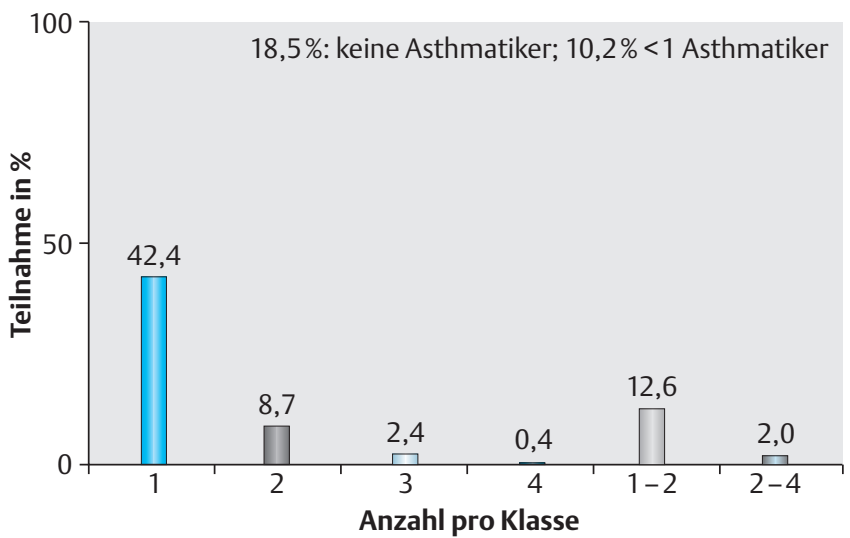

Abb. 2 Anzahl der Asthmatiker im Sportunterricht (mittlere Klassengröße 25-30 Schüler).

Bei möglicher Mehrfachnennung gaben 60,2\% die gleichberechtigte Teilnahme des erkrankten Kindes am Sportunterricht an, bei $37,8 \%$ (25,2 teilweise, $12,6 \%$ zeitweise) fand sich eine teiloder zeitweise Teilnahme und $13 \%$ nahmen überhaupt nicht am Sport teil (Abb. 3).

Ein Großteil der Asthmatiker erhielt die gleiche Benotung wie die gesunden Klassenkameraden (57,5\%), wobei der Unterricht aber nur bei 4,7\% einen speziellen, auf die Erkrankung abgestimmten, Aufbau aufwies. (Abb. 3 u. 4).

$59,8 \%$ der befragten Lehrer schrieben sich ein mittleres bis gutes Wissen, bei jedoch sehr breit gefächerter Quellenangabe, über die Erkrankung Asthma bronchiale zu (Abb.5). 


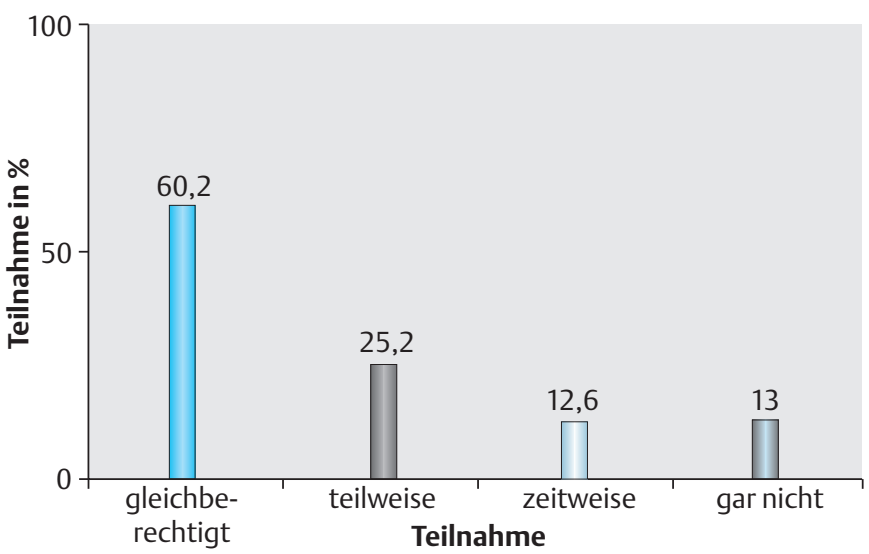

Abb. 3 Teilnahme der Asthmatiker am Sportunterricht.

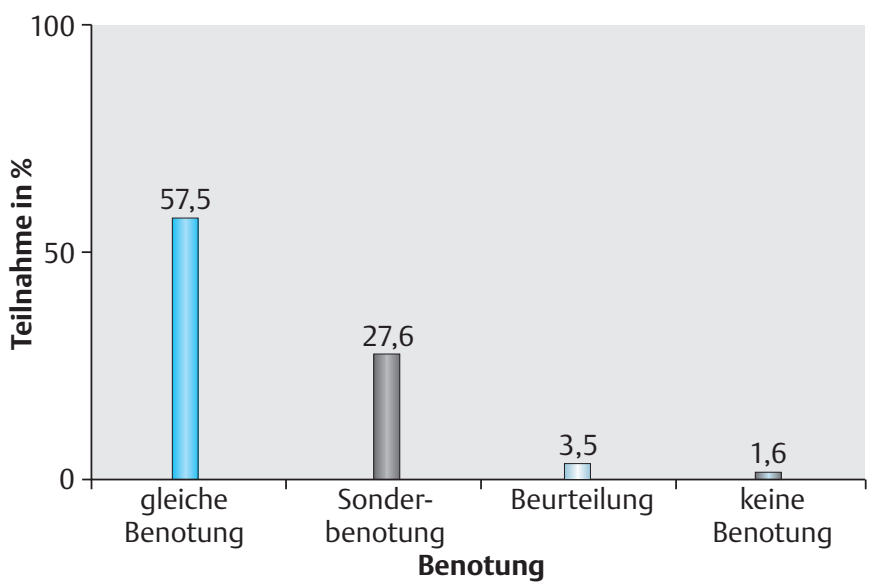

Abb. 4 Benotung des Schulsports.

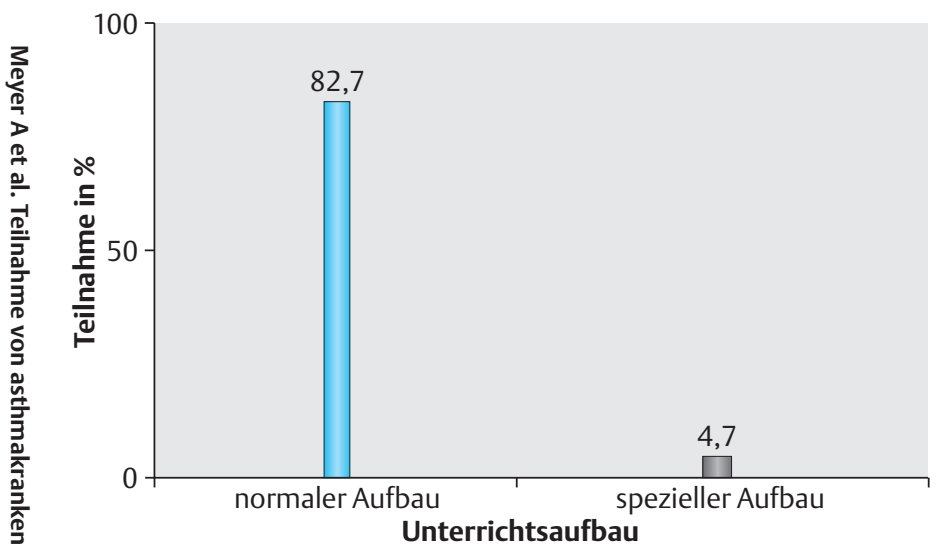

Abb. 5 Aufbau des Sportunterrichts.

Des Weiteren wurde untersucht, wie sich Alter, Geschlecht und Berufserfahrung der befragten Lehrer auf die Teilnahme asthmakranker Kinder am Schulsport auswirkten.

Von 254 zurückerhaltenen Fragebogen (111 Männer, 143 Frauen) war ein Großteil der Lehrer älter als 40 Jahre, wobei der Anteil der Frauen, bezogen auf die Berufsjahre sowie die Varianz der Ausbildung, deutlich überwog.

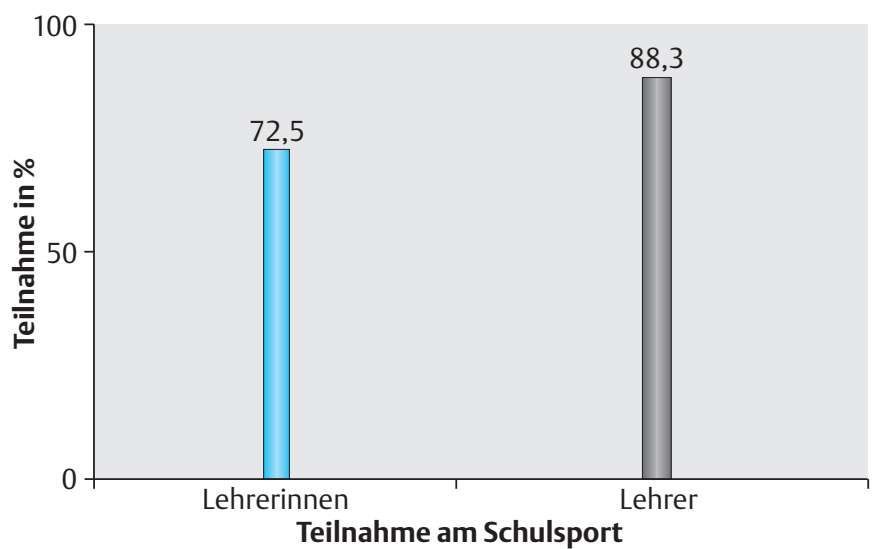

Abb. 6 Einfluss des Geschlechts auf die Teilnahme am Schulsport.

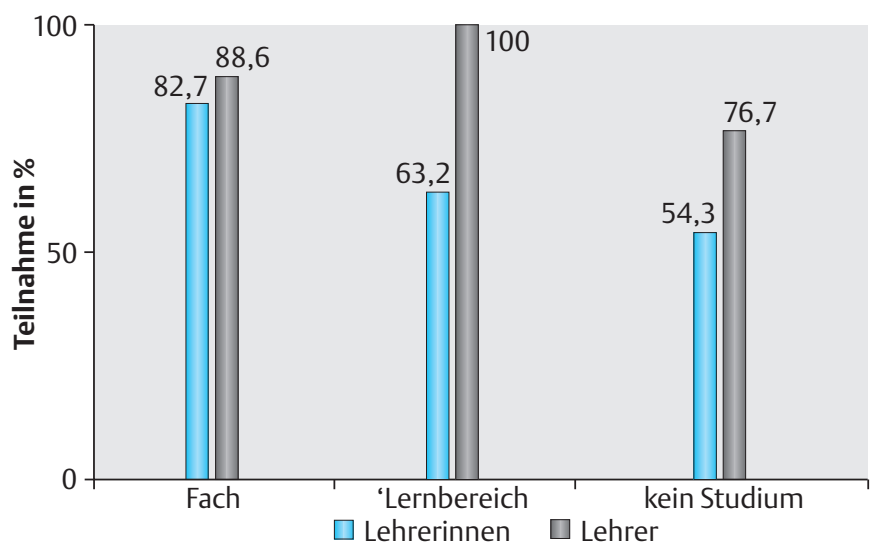

Abb. 7 Einfluss der Ausbildung und des Geschlechts auf die Teilnahme am Schulsport.

Während 88,3\% der Lehrer diese Schüler gleichberechtigt unterrichteten, waren es nur 72,5\% der Lehrerinnen. Es zeigt sich weiterhin, dass Lehrerinnen mit dem Lernbereich Sport (1.-4. Klasse) Asthmatiker insgesamt weniger am Sport teilnehmen lassen (63,2\%) als diejenigen mit der Fachausbildung Sport (5.-10./13. Klasse; 82,7\%). Dieses Verhältnis kehrt sich bei den männlichen Kollegen um: mit dem Lernbereich Sport fand sich eine 100\%ige Teilnahme gegenüber $88,6 \%$ bei einer sportlichen Fachausbildung und 76,7\% bei fehlender Ausbildung (Abb. 6 u. 7).

Es konnte weiterhin ein Unterschied der Teilnahme von Asthmatikern am Sport in Bezug auf die Berufserfahrung bei Frauen und Männern herausgestellt werden.

Bei Lehrerinnen mit bis zu 15-jähriger Berufserfahrung kommt es in $66,7 \%$ zur Sportteilnahme, bei mehr als 15-jähriger Berufserfahrung sogar zu $91,1 \%$.

Bei den männlichen Kollegen zeigt sich ein Verhältnis von 83,3\% zu 93,1\% (Abb. 8). 


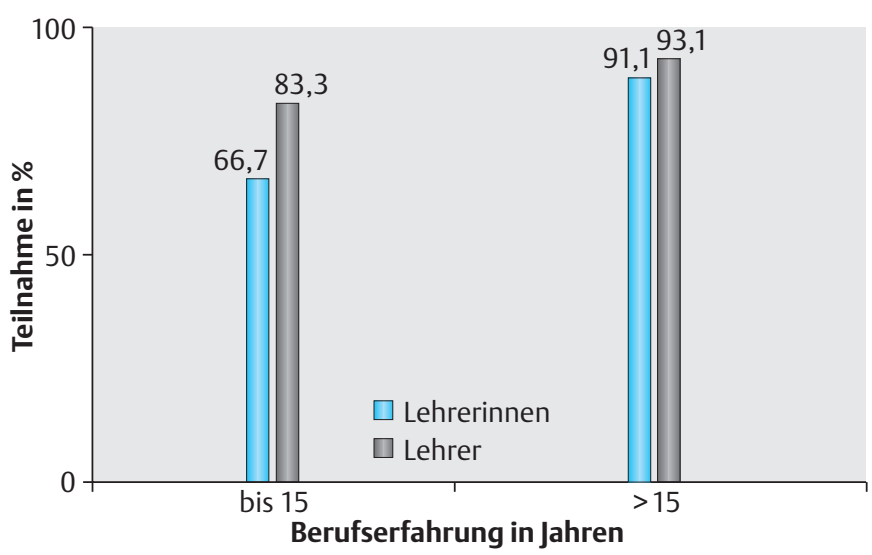

Abb. 8 Einfluss der Berufserfahrung und des Geschlechts auf die Teilnahme am Schulsport.

\section{Diskussion}

Da es sich beim Asthma bronchiale um die häufigste chronische Erkrankung im Kindes- und Jugendalter, bei weiter steigender Prävalenz, handelt, werden zunehmend auch Schule und Lehrer mit diesem Krankheitsbild konfrontiert.

Trotz nachgewiesener positiver Auswirkung von körperlicher Aktivität auf die Erkrankung Asthma bronchiale [14,15] wird dennoch ein Großteil der erkrankten Kinder nicht in den Sportunterricht integriert. Diese negative Einstellung gegenüber körperlicher Anstrengung beruht unter anderem darauf, dass $90 \%$ aller Asthmatiker auf körperliche Belastung mit einer Verengung der Bronchien reagieren [3]. Aktuelle Zahlen zur Teilnahme von asthmakranken Kindern am Schulsport liegen jedoch nicht vor. Dies veranlasste uns, die Teilnahme asthmakranker Kinder am Schulsport in Hamburg zu untersuchen.

Der Rücklauf der Studie erwies sich mit 65,8\% auswertbaren Fragebogen, bezogen auf allgemeine Bewertungsrichtlinien, als gut.

Auffällig war, dass in 42,4\% 1 Kind bzw. in 12,6\% 1 - 2 Kinder pro Klasse an einem Asthma bronchiale erkrankt waren, was einer Inzidenz von 3-5\% entspricht. Verglichen mit den Informationen der Behörde für Arbeit, Gesundheit und Soziales/Amt für Gesundheit in Hamburg (BAGS), ergaben sich bei Erhebungen zwischen 1990-97 in den 4. und 8. Klassen mit einer Inzidenz von im Mittel 3,8\% bzw. 4,4\% ähnliche Zahlen. In der deutschen Literatur werden hingegen Zahlen zwischen 5 und $15 \%$ beschrieben [17], woraus zu schließen ist, dass offensichtlich in Hamburg nicht alle Kinder, die an einem Asthma bronchiale erkrankt sind, auch als solche identifiziert werden. Dies trifft wahrscheinlich für die Kinder zu, die an einem intermittierenden oder leichtgradigen Asthma erkrankt sind. Die häufig mangelnde Identifikation dieser Erkrankung belegte auch eine an 3300 Schülern aus 12 verschiedenen Mittelschulen durchgeführte Screening-Untersuchung in den USA [18].

Weitere Diskrepanzen ergaben sich zwischen der Art und Häufigkeit der sportlichen Beteiligung auf der einen Seite und dem tatsächlichen Wissensstand der Lehrer auf der anderen Seite.
Es nehmen zwar 74,4\% am Sport teil, aber nur $60 \%$ werden auch gleichberechtigt integriert. Diese für das Hamburger Stadtgebiet ermittelten Angaben über die Teilnahme entsprechen in etwa denen einer Untersuchung von Lecheler von 1991, die besagt, dass 30\% der Asthmatiker vom Schulsport befreit sind, wobei es sich hier allerdings bereits um ein stark vorselektioniertes Patientenkollekiv handelt [16]. In weiteren Studien konnte aber gezeigt werden, dass sich eine sportliche Beteiligung asthmakranker Kinder sowohl positiv auf physische als auch auf psychische Faktoren auswirkt und aus diesem Grund von großer Bedeutung für die erkrankten Kinder und Jugendlichen ist. Es wurden eine Verbesserung der Vitalkapazität, eine bessere Belastbarkeit bei insgesamt weniger Anfällen [15], eine effektivere Sauerstoffaufnahme, sowie eine sinkende Herzbelastung beobachtet, so dass durch regelmäßiges Training der Bewegungsspielraum betroffener Kinder vergrößert werden kann [19]. Bei sinkender Beschwerdesymptomatik und geringem Medikamentenverbrauch können Asthmatiker sogar zum Teil gute bis sehr gute Leistungen auf Wettkampfebene erbringen [5,15]. Interessant ist, dass die kardiopulmonale Belastbarkeit des Patienten nicht unbedingt mit dem Schweregrad der Erkrankung [12], der Häufigkeit von Krankenhausaufenthalten, der Steroidtherapie oder dem Auftreten schwerer Anfälle verknüpft ist, sondern oft mit der psychosozialen Komponente, dem Befinden und der Einstellung gegenüber sportlicher Betätigung verbunden ist [20].

Eine Studie von Brook [21] verdeutlichte sogar, dass die Haltung gesunder und an Asthma bronchiale erkrankter Kinder und Jugendlicher nahezu gleich sei, was auch andere Studien gezeigt haben [22] und dass Asthmatiker, ausgehend von ihrer positiven Einstellung, gewillt sind, gleichberechtigt am Sportunterricht teilzunehmen. Diese Ergebnisse werden durch eine Studie aus Skandinavien gestützt, die zeigen konnte, dass in einem Alter zwischen 7 und 16 Jahren kein Unterschied in der körperlichen Aktivität eines Gesunden und eines Asthmatikers besteht [15].

Ein Ausschluss der asthmakranken Kinder vom Sportunterricht scheint also in keinem Fall gerechtfertigt, zumal hierdurch irreversible schulische und Entwicklungs-Defizite hervorgebracht werden können [10,23].

Berücksichtigt werden muss allerdings die Tatsache, dass eine unvermittelt einsetzende körperliche Belastung (5 Runden laufen um den Sportplatz) zu einer ausgeprägten Obstruktion der Atemwege führen kann [9].

Aus diesem Grund ist es für den Sportlehrer von Bedeutung zum einen Kenntnisse bezüglich der Prämedikation und gegebenenfalls der Intervention bei einem Asthmaanfall zu haben als auch bestimmte Regeln für die Durchführung einer Sportstunde beim asthmakranken Kind einzuhalten. Hierzu gehören z. B. eine entsprechende Aufwärmphase, ein Intervalltraining, Auswahl geeigneter Sportarten, gegebenenfalls besondere Vorsicht bei Wettkampfsportarten sowie eine entsprechende Abklingphase bei insgesamt möglichst submaximalen Belastungen [16].

In unserer Studie zeigte sich jedoch, dass bei 82,7\% der Lehrer kein spezieller Unterrichtsaufbau angegeben wurde, woraus geschlossen werden kann, dass trotz positiver Selbsteinschätzung bezüglich der Kenntnisse der Erkrankung offensichtlich ein deutlich schlechterer Informations- und Wissensstand zugrunde liegt. 
Dieses Ergebnis deckt sich mit vielen anderen Studien [8,24,25], wobei zum Teil auch in der Selbsteinschätzung Wissensdefizite bis zu $93 \%$ in den unterschiedlichen Bereichen vermerkt werden [26-28]. Die in diesem Zusammenhang aktuellste Studie mit über 400 Fragebogen zur Wissenserfassung zeigte, dass zum Teil gravierende Defizite in den Bereichen Erfahrung mit Asthma, Allgemeinwissen, Symptomatik, Auslöser, Therapie und Asthmaberücksichtigung beim Sport bestehen und die Lehrer häufig mit der Betreuung des asthmakranken Kindes überfordert sind [8] oder diese gar nicht erst identifizieren können. In weiteren Studien wird deutlich, dass bei relativ gutem medizinischen Basiswissen über Asthma bronchiale erhebliche Defizite bezüglich möglicher Therapieoptionen bestehen und mehr als $50 \%$ der befragten Lehrkörper die Bedeutung präventiver Maßnahmen unterschätzen $[29,30]$.

Eine in Australien durchgeführte Studie zeigte auch, dass ein weiteres großes Problem der Umgang mit entsprechender Medikation zu sein scheint, da das Mitbringen eigener Medikation entweder in einigen Ländern verboten ist [31] oder aber Medikamente ohne Hintergrundwissen dennoch verabreicht werden [23].

Die Tatsache, dass eine effektive medizinische Therapie, Supervision der Sportlehrer sowie Ergreifen von Präventivmaßnahmen das Risiko für eine belastungsinduzierte Obstruktion der Bronchien beim Sportunterricht minimieren können [32], zeigt, dass eine Schulung von Lehrern notwendig ist.

So sind bereits 2-stündige Seminare oder Workshops in der Lage, Wissen zu verbessern [4]. Besonders erwähnenswert ist die Studie von Szczepanski, die eine Schulung zum Thema Asthma bronchiale für Lehrerkollegien an insgesamt 40 Schulen untersuchte. Es wurden 3-stündige Seminare in Form eines interaktiven Gespräches sowie zahlreiche praktische Übungen angeboten. Hierbei wurde das gesamte Kollegium in die Fortbildung eingeschlossen und konnte auf den gleichen Wissensstand gebracht werden. Schulinterne Seminare scheinen hierbei sinnvoller als überregionale Veranstaltungen zu sein. Aus Sicht der Teilnehmer waren die Seminarinhalte sowie die Umsetzung eines Notfallplanes in den Schulalltag integrierbar.

Auch auf die Möglichkeit eines medizinischen Basistrainings mit regelmäßig ergänzenden Seminaren innerhalb der Lehrerausbildung sei in diesem Zusammenhang hingewiesen [33].

Fest steht, dass der richtige Umgang mit asthmakranken Kindern die richtige Einschätzung des Kindes bezüglich der Schwere der Erkrankung und der körperlichen Leistungsfähigkeit erfordert [34]. Dies setzt eine gute Kommunikation zwischen Betroffenen, Eltern, Lehrern und Ärzten voraus, was auch andere Studienergebnisse bestätigen [25].

Die hier vorliegende Erhebung in Hamburg hat ergeben, dass offensichtlich auch das Geschlecht und die vorliegende Berufserfahrung einen Einfluss auf den Umgang mit Asthmatikern haben.

Erkrankte Kinder wurden häufiger von Lehrern als von Lehrerinnen gleichberechtigt in den Sportunterricht integriert ( $\mathrm{m}: 88,3 \%$, w: 72,5\%). Bei Lehrkräften mit Sport als Ausbildungsfach war eine $88,6 / 82,7 \%$ ige Teilnahme ( $\mathrm{m} / \mathrm{w})$, mit Sport als Lernbereich eine 100/63,2\%ige Teilnahme $(\mathrm{m} / \mathrm{w})$ zu verzeichnen und bei mehr als 15-jähriger Berufserfahrung wurden die erkrankten Kinder zu 93,1/91,3\% $(\mathrm{m} / \mathrm{w})$ gegenüber $83,6 / 66,7 \%(\mathrm{~m} / \mathrm{w})$ bei geringer Berufserfahrung integriert.

Somit scheint ein Wissensaustausch innerhalb eines meist heterogenen Kollegiums wichtig zu sein, was durch die schulinterne Fortbildung gefördert würde. Voraussetzung für eine bessere Integration von Kindern mit Asthma ist die exakte Diagnose der Erkrankung. Hier sind vor allem Ärzte und Eltern gefordert. Um die Situation der an Asthma erkrankten Kinder in der Schule zu verbessern, ist unserer Ansicht nach erforderlich, das Basiswissen über diese häufigste chronische Erkrankung im Kindesalter in die Aus- und Weiterbildung der Lehrer zu integrieren. Dies sollte im Studium beginnen. Das Wissen muss jedoch auch vor Ort in der Schule kontinuierlich aktualisiert werden. Entsprechend weitergebildete Lehrer sind dann nicht nur in der Lage, Asthmatiker besser in den Schulalltag zu integrieren, sondern können auch den Eltern wichtige Hinweise geben, die zur Diagnose und besseren Behandlung der Erkrankung beitragen.

\section{Literatur}

${ }^{1}$ Wijst N. Epidemiologie von Asthma im Kindesalter im internationalen Vergleich. Allergologie 1996; 19: $234-240$

2 Baumann A, Mitchell CA, Henry RL. Asthma morbidity in Australia: An epidemiological study. Med J Aust 1992; 156: 827-831

${ }^{3}$ Skjonsberg O, Clench-As J, Leeggaard J et al. Prevalence of bronchial asthma in school children in Oslo, Norway. Allergy 1995; 50: 806-810

${ }^{4}$ Hill R, Williams J, Tattersfield A et al. Change in use of asthma as a diagnostic label for wheezing illness in schoolchildren. B M J 1989; 299; 898

${ }^{5}$ Higgins L. Allergies and asthma in the school setting. Immunology and allergy clinics of North America 1999; 19: 177 - 189

${ }^{6}$ Berdel D, Reinhardt D, Hofmann D et al. Therapieempfehlung der Gesellschaft Pädiatrische Pneumologie zur Behandlung des Asthma bronchiale bei Kindern und Jugendlichen. Monatsschr Kinderheilkd 1998; 146: $492-497$

${ }^{7}$ Peat J, van den Berg R, Grenn W et al. Changing prevalence of asthma in Australian children. B M J 1994; 308: 1591 - 1596

${ }^{8}$ Szepanski R, Brockmann G, Friede G. Asthmaschulung für Lehrer Notwendigkeit und Möglichkeiten. Pneumologie 2001; 55: 512 - 519

${ }^{9}$ Goldberg B. Children, sports and chronic disease. The physcian and sportsmedicine 1990; 18 (10): $45-56$

${ }^{10}$ Lecheler J, Gauer S. Schuldefizite chronisch asthmakranker Kinder und Jugendlicher. Monatsschr Kinderheilkd 1991; 139: 69-72

11 Levy M, Bell L. General practice audit of asthma in children. B M J 1984; 289: 1115 - 1116

12 Juniper E. Quality of life in adults and children with asthma and rhinitis. Allergy 1997; 52: $971-977$

${ }^{13}$ Christiansen M, Lavigne J, Lerner C. Psychosocial aspects of compliance in children and adolescents with asthma. J Dev Behav Pediatr 1989; 10: $75-90$

${ }^{14}$ Brook U, Heim M. A pilot study to investigate whether sport influences psychosocial parameters in the personality of asthmatic children. Fam Pract 1991; 8 (3): 1 -3

${ }^{15}$ Nystad W. The physical activity level in children with asthma based on a survey among 7 -16-year-old school children. Scand J Med Sci Sports 1997; 7: $331-335$

${ }^{16}$ Lecheler J. Sport bei asthmakranken Kindern und Jugendlichen - Risiko oder Chance? Öff Gesundh-Wes 1991; 53: 486-489

17 von Mutius E, Nowak D. Epidemiologische und sozialmedizinische Aspekte des Asthma bronchiale im Kindes- und Erwachsenenalter. In: Petermann F, Warschburger P (eds). Asthma bronchiale. Göttingen: Hogrefe Verlag, 2000

18 Voelker R. Quick uptakes: Screen for Asthma at school. JAMA 2000; 284: 2585

${ }^{19}$ Völker M. Ergebnisse eines sechswöchigen Intervalltrainings bei asthmakranken Jugendlichen [Dissertation]. 1986 
${ }^{20}$ Bender B, Belleau L, Fukuhara J et al. Psychomotor adaption in children with severe chronic asthma. Pediatrics 1987; 79: 723 - 727

${ }^{21}$ Brook U, Stein D, Alkalay Y. The attitude of asthmatic and nonasthmatic adolescents toward gymnastic lessons at school. J Asthma 1994; 31 (3): $171-175$

22 Weston R, Macfarlane D, Hopkins W. Physical activity of asthmatic and nonasthmatic children. J Asthma 1989; 26: 279-286

${ }^{23}$ Freudenberg N, Feldmann C, Clark N. The impact of bronchial asthma on school attendance and performance. J Sch Health 1980; 50: 522

${ }^{24}$ French D, Carroll A. Western Australian primary school teachers' knowledge about childhood asthma and its management. J Asthma 1997; 34(6): 469-475

25 Pin I, Guerin-Develay S, Cans C, Vivier S et al. Étude transversale de l'intégration scolaire des enfants asthmatiques issus dùn échantillon de la population générale. Arch pédiatr 2000; 7: 817-824

${ }^{26}$ Brookes J, Jones K. Schoolteachers' perceptions and knowledge of asthma in primary school children. Br J Gen Pract 1992; 42: $504-507$

${ }^{27}$ Atchison J, Cuskelly M. Educating teachers about asthma. J Asthma 1994; 31: 269-276

28 Stollhofer , B , Lahrmann H, Zwick H. Erhebung des Wissensstandes von Wiener VolksschullehrerInnen über Asthma bronchiale bei Kindern. Pneumologie 1998; 52: 406-411

${ }^{29}$ Bevis M, Taylor B. What do school teachers know about asthma? Arch Dis Child 1990; 65: 622-625

${ }^{30}$ Brookes J, Jones K. Schoolteachers' perceptions and knowledge of asthma in primary schoolchildren. Br J Gen Pract 1992; 42: 504-507

${ }^{31}$ Regis D. Asthma in the classroom: What can be done? What should be done? Exeter, Schools Health Education Unit), 1994

32 Croft D, Lloyd B. Asthma spoils sport for too many children. Practitioner 1989; 233: 969 - 971

${ }^{33}$ Bowen C. Education teachers in children's illnesses: a study. Nurs Stand 1996; 10: $33-36$

${ }^{34}$ Rifat S, Ruffin M, Gorenflo D. Disqualifying criteria in a preparticipation sports evaluation. J Fam Pract 1995; 41 (1): $42-50$

Asthma und Schulsport - ein möglicher Versicherungsfall?

Ein Beitrag der Landesunfallkasse Hamburg

Dipl.-Ing. Hans-Joachim Chilla, Präventionsabteilung,

Referat Schulen und Hochschulen

Aus der Sicht der Unfallverhütung und der Förderung der Sicherheit und des Gesundheitsschutzes sehen wir als Träger der gesetzlichen Unfallversicherung in Hamburg die Notwendigkeit, der Bewegung in der Schule - und hier insbesondere im Schulsport - wieder einen höheren Stellenwert einzuräumen. Spiel und Sport fördern nicht nur langfristig die Bewegungssicherheit, sondern haben auch kurzfristig positive Auswirkungen auf das Unfallgeschehen in der Schule. Gesundheitliche Beeinträchtigungen bei Schülerinnen und Schülern müssen diesem Ziel nicht zwangsläufig entgegenstehen, wenn die Verantwortlichen in der Schule einfachste Überlegungen anstellen und sich präventives, d.h. umsichtiges und vorausschauendes Handeln zu eigen machen (siehe folgenden Kasten).

\begin{tabular}{|c|c|}
\hline $\begin{array}{l}\text { Lehrerinnen und Lehrer sollten } \\
\text { - sich fragen } \\
\text { - wissen } \\
\text { - tun }\end{array}$ & $\begin{array}{l}\text { - Habe ich mein Wissen als Ersthelfer regel- } \\
\text { mäßig - alle } 2 \text { Jahre - trainiert? } \\
\text { - Kenne ich den Gesundheitszustand meiner } \\
\text { Schülerinnen und Schüler? } \\
\text { - Habe ich in Zweifelsfällen die Eltern nach } \\
\text { den individuellen Risiken ihrer Kinder } \\
\text { gefragt? }\end{array}$ \\
\hline $\begin{array}{l}\text { Erkrankungen müssen bekannt } \\
\text { sein bei } \\
\text { - Sport allgemein } \\
\text { - Schwimmen } \\
\text { - Klassenfahrten }\end{array}$ & $\begin{array}{l}\rightarrow \text { Allergien } \\
\rightarrow \text { Zuckerkrankheit } \\
\rightarrow \text { Asthma } \\
\rightarrow \text { Epilepsie } \\
\rightarrow \text { Blutungsneigung } \\
\rightarrow \text { Ohrenerkrankungen } \\
\rightarrow \text { Behinderungen }\end{array}$ \\
\hline $\begin{array}{l}\text { Notfallmedikamente gehören } \\
\text { nicht in den Verbandschrank }\end{array}$ & $\begin{array}{l}\text { - Sind die mir bekannten risikobelasteten } \\
\text { Schülerinnen und Schüler in Behandlung } \\
\text { und derzeit anfallsfrei? } \\
\text { - Habe ich mit den Eltern, den Schülerinnen } \\
\text { und Schülern Regelungen für das Verabrei- } \\
\text { chen von Notfallmedikamenten getroffen? } \\
\text { - Ist gewährleistet, dass Schülerinnen und } \\
\text { Schüler ihre Notfallmedikamente immer } \\
\text { bei sich haben? } \\
\text { - Sind Kolleginnen und Kollegen, die mich } \\
\text { vertreten sollen, über meine risikobelaste- } \\
\text { ten Schülerinnen und Schüler informiert? }\end{array}$ \\
\hline $\begin{array}{l}\text { Entscheidungen nach bestem } \\
\text { Wissen und Gewissen - weder } \\
\text { fahrlässig noch grob fahrlässig }\end{array}$ & $\begin{array}{l}\text { Sie haben alle denkbaren Überlegungen an- } \\
\text { gestellt. Sie haben sich - auch im Einverneh- } \\
\text { men mit den Eltern - für eine Teilnahme ihrer } \\
\text { risikobelasteten Schülerinnen und Schüler bei } \\
\text { allen sportlichen Übungen entschieden: Ein } \\
\text { guter Entschluss - insbesondere für deren } \\
\text { Gesundheit. Ihre Einschätzung ist gerechtfer- } \\
\text { tigt, selbst wenn sie zu einem Unfall führen } \\
\text { würde. }\end{array}$ \\
\hline $\begin{array}{l}\text { Verabreichen von Notfallmedi- } \\
\text { kamenten ist erste Hilfe }\end{array}$ & $\begin{array}{l}\text { Sie verabreichen Notfallmedikamente und } \\
\text { leisten damit - ihren Fähigkeiten und den } \\
\text { Umständen entsprechend - erste Hilfe. Sie } \\
\text { brauchen dann grundsätzlich weder mit zivil- } \\
\text { rechtlichen noch mit strafrechtlichen Konse- } \\
\text { quenzen zu rechnen, die sich nachteilig für sie } \\
\text { auswirken. }\end{array}$ \\
\hline $\begin{array}{l}\text { Weitergehende medizinische } \\
\text { Beratung - kostenlos für sie }\end{array}$ & $\begin{array}{l}\text { Unser Angebot: Im Team der Präventionsab- } \\
\text { teilung der Landesunfallkasse Hamburg berät } \\
\text { sie ein Facharzt für Arbeitsmedizin sowie Lun- } \\
\text { genheilkunde und Allergologie, Herr Dr. med. } \\
\text { Andreas Dittmann, Tel. 040-27153226. }\end{array}$ \\
\hline
\end{tabular}

Lehrerinnen und Lehrer sollten

- sich fragen

- wissen

(1)

Erkrankungen müssen bekannt sein bei

Sport allgemein

- Schwimmen

- Klassenfahrten

Notfallmedikamente gehören nicht in den Verbandschrank

Wissen und Gewissen - weder

fahrlässig noch grob fahrlässig

Verabreichen von Notfallmedikamenten ist erste Hilfe 